\title{
SURFACTANTS IN KLODNICA RIVER (KATOWICE, POLAND). PART II. QUATERNARY AMMONIUM COMPOUNDS
}

\author{
OBECNOŚĆ ZWIĄZKÓW POWIERZCHNIOWO CZYNNYCH W RZECE \\ KŁODNICY (KATOWICE; POLSKA) CZEŚ́́ II. CZWARTORZĘDOWE \\ ZWIĄZKI AMONIOWE
}

\begin{abstract}
The paper presents methods of determination of analytes of the cation group (alkyl benzyl dimethyl ammonium (BDDA-C12-C16), alkyl trimethyl ammonium (TMA), hexadecyl piridinium (HP)) in surface water and bottom sediment samples. In the sample preparation phase the solid phase extraction (SPE) or accelerated solvent extraction/ultrasound assisted extraction (ASE/UAE)-SPE technique was used and in the identification phase and quantitative determination of analytes phase - ion chromatography technique (combined with a conductivity detector (CD)). The determined concentrations were in the range below the determined method detection limit (MDL) or method quantification limit (MQL) figures up to $0.142 \pm 0.023 \mathrm{mg} / \mathrm{dm}^{3}$ or $2014 \pm 10 \mu \mathrm{g} / \mathrm{kg}$ (liquid and solid samples, respectively). Comparing concentrations of individual analytes found in liquid and solid environmental samples we may notice that surfactants containing a shorter alkyl chain in their molecules were found in higher concentrations in liquid samples (hydrophobicity increasing with the chain length).
\end{abstract}

Keywords: surface-active substances, surface water, industrial area, quaternary ammonium compounds

\section{Introduction}

Surface active compounds (SAC), due to their specific physical and chemical properties are widely used in industry and households. The use of different types of surfactants in many areas of human activity contributes to their introduction into individual elements of the environment. It is important to determine if these compounds have a negative impact on animals and plants (particularly on aquatic organisms). Some surface active compounds are low biodegradable and products of their degradation are more toxic to living organisms than the original compounds or they exhibit an endocrine activity [1].

\footnotetext{
${ }^{1}$ Faculty of Earth Sciences, University of Silesia, ul. Będzińska 60, 41-200 Sosnowiec, Poland, phone +48 3236894 37, +48 3236892 13, email: marek.ruman@us.edu.pl, slawomir.pytel@us.edu.pl

${ }^{2}$ Department of Environmental Toxicology, Faculty of Health Sciences with Subfaculty of Nursing and Institute of Maritime and Tropical Medicine, Medical University of Gdansk, ul. Dębowa 23A, 80-204 Gdańsk, Poland, phone +4858349 19 35, email: ewa.olkowska@ gumed.edu.pl

${ }^{3}$ Department of Analytical Chemistry, Faculty of Chemistry, Gdansk University of Technology, ul. G. Narutowicza 11/12, 80-233 Gdańsk, Poland, phone +48 58347 21 10, email: zanpolko@pg.gda.pl

*Corresponding author: zanpolko@pg.gda.pl
} 
Cationic surfactants have biostatic or biocidal properties and they are used in various bactericidal and fungicidal products that limit the development of microorganisms (bacteria, yeast, fungi) or kill them [2]. Moreover, cationic compounds, because their particles are positively charged, have the ability to undergo sorption processes on negatively charged surfaces of solids (e.g. bottom deposits, soil, metals, plastics and cell membranes), what increases the possibility of accumulation or bioaccumulation processes [3].

In the case of quaternary ammonium salts (QAC), which are strong biostatic and biocidal agents [4], the length of alkyl chain present in their particle influences the environmental fate of this group of cationic surfactants $[5,6]$. The lower the number of methyl groups the lower the ability to undergo aerobic biodegradation by QAC compounds, because methyl groups are substituted with benzyl groups or long alkyl groups (e.g. $\mathrm{R}_{4} \mathrm{~N}^{+}<\mathrm{R}_{3} \mathrm{MeN}^{+}<\mathrm{R}_{2} \mathrm{Me}_{2} \mathrm{~N}^{+}<\mathrm{RMe}_{3} \mathrm{~N}^{+}<\mathrm{Me}_{4} \mathrm{~N}^{+}$; where: Me - methyl group) [7, 8].

Surfactants with a cationic character of the polar part of the particle, usually contain a tertiary or quaternary nitrogen atom (positively charged) bound with hydrocarbon chains (alkyl or alkyl benzyl). Moreover, a nitrogen atom may occur in a heterocyclic ring and the non-polar part of such surface-active compounds may be bound with the nitrogen atom or with another atom found in the ring.

Quaternary ammonium compounds are cationic surfactants often used by people during various forms of activity. QAC are commonly used as surfactants in drilling muds, fabric softeners and as biocides in antiseptics and disinfectants $[9,10]$.

Particles of such compounds are built of a quaternary nitrogen atom with four hydrocarbon substituents attached by a covalent bond [11]. Table 1 presents structural formulas of selected compounds from the cationic surfactants group.

Table 1

Structural formulas of selected compounds from the group of cationic surfactants

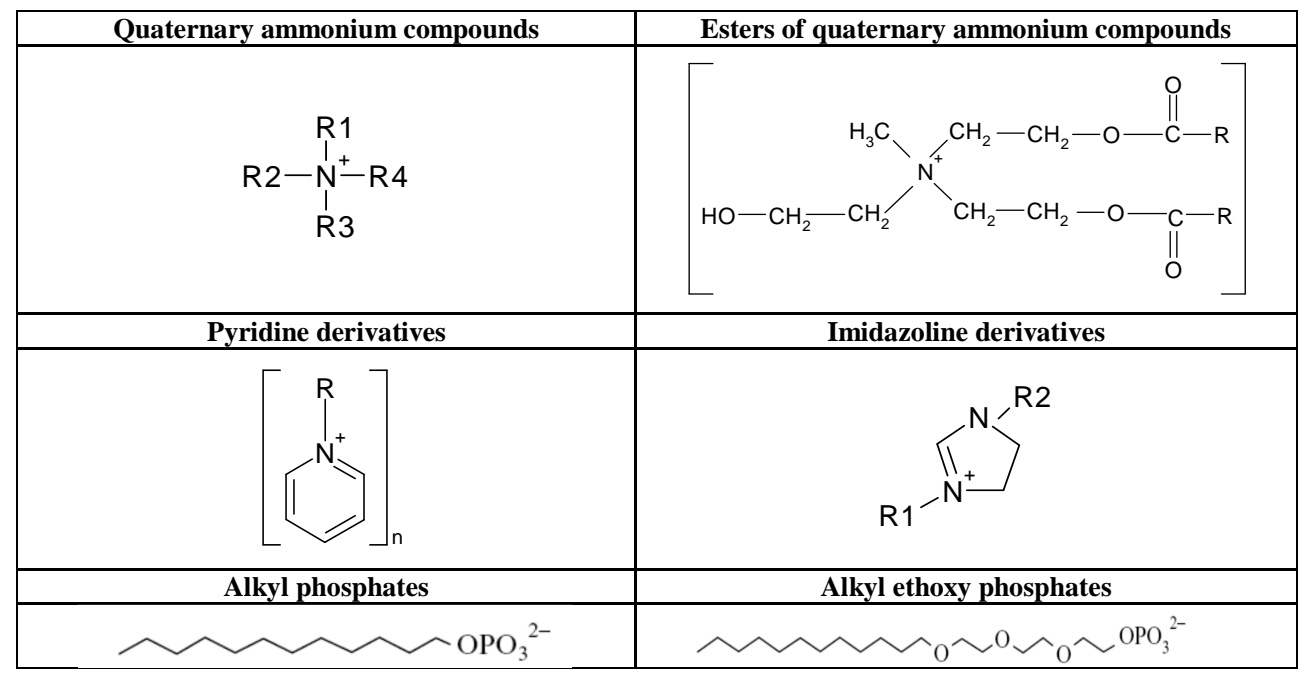

The analysis of literature makes it possible to state that surfactants may have a varied influence on living organisms (Table 2). 
Information on toxicity of cationic surfactants on living organisms

\begin{tabular}{|c|c|c|c|c|}
\hline $\begin{array}{l}\text { Surfactant } \\
\text { (acronym) }\end{array}$ & Bioindicator & Toxicity factor & $\begin{array}{c}\text { Concentration } \\
\text { range }\left[\mathrm{mg} / \mathrm{dm}^{3}\right]\end{array}$ & References \\
\hline \multirow{2}{*}{$\begin{array}{l}\text { TMAC - Trimethyl } \\
\text { ammonium chloride }\end{array}$} & Dunaliella salina & $E C_{50} / 24 \mathrm{~h}$ & 0.79 & {$[12]$} \\
\hline & Daphnia magna & $I C_{50} / 24 \mathrm{~h}$ & $0.13-0.38$ & [7] \\
\hline \multirow{2}{*}{$\begin{array}{c}\text { BDMAC - Benzyl } \\
\text { dimethyl ammonium } \\
\text { chloride }\end{array}$} & Dunaliella salina & $E C_{50} / 24 \mathrm{~h}$ & 1.3 & {$[12]$} \\
\hline & Daphnia magna & $I C_{50} / 24 \mathrm{~h}$ & $0.13-0.22$ & [7] \\
\hline \multirow{3}{*}{$\begin{array}{l}\text { DTDMAC - Ditallow } \\
\text { dimethyl ammonium } \\
\text { chloride }\end{array}$} & Daphnia magna & $L C_{50} / 48 \mathrm{~h}$ & 0.49 & [13] \\
\hline & Goldfish (Carassius auratus) & \multirow{2}{*}{${ }^{*} E C_{50} / 48 \mathrm{~h}$} & 2.37 & \multirow{2}{*}{ [14] } \\
\hline & Rainbow trout (Salmo gairdneri) & & 0.74 & \\
\hline
\end{tabular}

$E C_{50}$ - effective concentration, ${ }^{*} E C_{50}$ - immobilization effective concentration, $I C_{50}$ - inhibitory concentration, $L C_{50}$ - lethal concentration

In the carried out research environmental samples collected in the Klodnica catchment were used. This river was selected as the proper ecosystem to verify the developed analytical solutions concerning determination of selected analytes from the group of cationic surfactants in environmental samples. The river catchment is located in a highly urbanised and industrialised area described as the Upper Silesian Industrial Region. The location of the region is connected mainly with the occurrence of mineral deposits, what as a consequence led to the necessity of development of the required infrastructure making possible its exploitation and advancing urbanisation. In the Upper Silesian Industrial Region there exist such manifestations of human activity as: hard coal deep mining; iron and steel industry; power plants and combined heat and power (CHP) plants; automotive industry, chemical industry (manufacture of detergents, etc.); direct discharge of municipal wastewater into the river; air, land and water transport $[15,16]$.

On the basis of long-term research it has been established that the Klodnica is highly polluted throughout its nearly whole length due to the emission of various chemical compounds with treated effluents or their discharge without previous treatment. Additionally, pollutants may be introduced into surface water with atmospheric precipitation, when they are washed into the sewerage system through highly urbanised/industrialised areas and then reach the river network. On certain stages of circulation in the environment rainwater is enriched with different chemical compounds (e.g. organic compounds, heavy metals) and high quantities of suspension that may be directed to surface water $[15,16]$.

The above aspects confirm the possibility of pollution of the Klodnica's ecosystem with surfactants and comprehensive research in this area is justified. The river catchment may be a real system, where the process of verification of the developed analytical methodologies can be carried out and the degree of pollution of certain elements of the ecosystem (surface water and bottom sediments) with different surfactants (e.g. not included in legal regulations concerning the monitoring of aquatic ecosystems) can be determined. 


\section{Experimental procedures}

\section{Sampling site and sampling method}

During the carried out research environmental samples collected in the Klodnica catchment were used. The Klodnica catchment is located in the western part of Silesian Voivodeship and it is the right tributary of the Oder. It flows through cities like: Katowice, Ruda Slaska, Mikolow, Ornontowice, Gieraltowice, Zabrze, Bytom, Chorzow and Gliwice (Fig. 1). Detailed information about sampling sites is presented in the first part of the publication $[17,18]$.

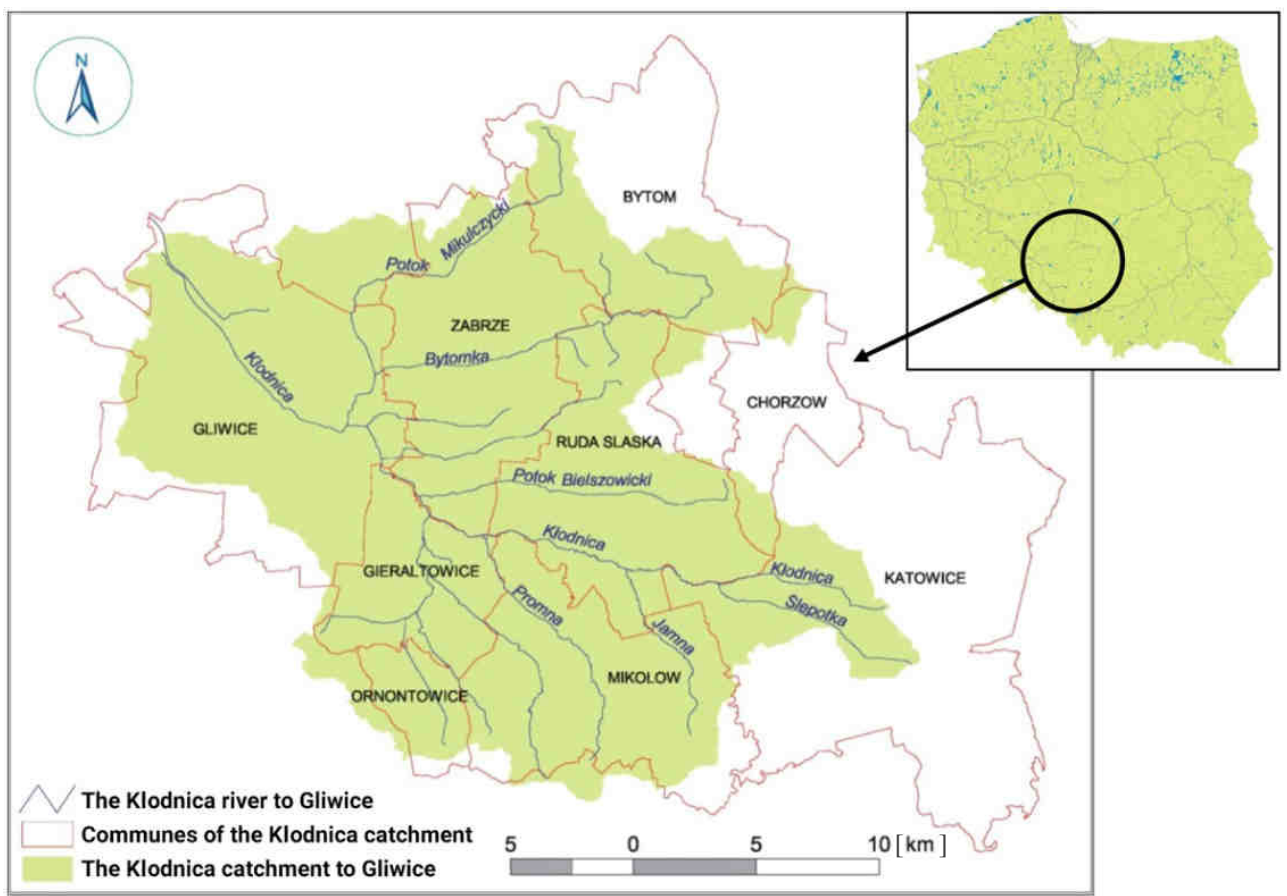

Fig. 1. Location of the Klodnica catchment (authors' own elaboration on the basis of [17])

\section{Analytical laboratory methods}

Table 3 contains detailed information about selected analytes from the surfactants group, like molar masses, chemical structure and data about toxicity of the determined compounds.

Ion chromatograph equipped with a conductometric detector was used at the stage of detection, identification and quantitative determination of content levels of certain surfactants in especially prepared solutions of standard substances and extracts of environmental samples [19, 20]. Optimised operating parameters of a chromatographic system during analyses are presented in Table 4. 
Basic information about the properties of selected analytes from the surfactants group

\begin{tabular}{|c|c|c|c|c|c|}
\hline $\begin{array}{l}\text { Name/chemical } \\
\text { formula }\end{array}$ & $\begin{array}{c}\text { Molar } \\
\text { mass } M \\
{[\mathrm{~g} / \mathrm{mol}]}\end{array}$ & $\begin{array}{l}\text { Structural } \\
\text { formula }\end{array}$ & $\begin{array}{c}\text { Harmful effects } \\
\text { on humans }\end{array}$ & Toxicity to animals & References \\
\hline $\begin{array}{c}\text { Dodecyl } \\
\text { benzyl dimethyl } \\
\text { ammonium } \\
\text { chloride } \\
\mathrm{C}_{21} \mathrm{H}_{38} \mathrm{NCl}\end{array}$ & 339 & $\begin{array}{c}\mathrm{CH}_{2}-\mathrm{C}_{6} \mathrm{H}_{5} \\
\mathrm{CH}^{+}-\mathrm{CH}_{3} \\
\mathrm{R}=\mathrm{CH}_{2}\left(\mathrm{CH}_{2}\right)_{12} \mathrm{CH}_{3}\end{array}$ & \multirow{3}{*}{$\begin{array}{l}\text { Causes burns of: } \\
\text { - } \text { eyes } \\
\text { - } \text { skin } \\
\text { - respiratory } \\
\text { system }\end{array}$} & $\begin{array}{c}\text { Rats: } L D_{50}=400 \mathrm{mg} / \mathrm{kg} \\
\quad \text { (orally) } \\
\text { Extremely toxic to aquatic } \\
\text { organisms }\end{array}$ & {$[21,22]$} \\
\hline $\begin{array}{c}\text { Tetradecyl } \\
\text { benzyl dimethyl } \\
\text { ammonium chloride } \\
\mathrm{C}_{23} \mathrm{H}_{42} \mathrm{NCl}\end{array}$ & 368 & $\begin{array}{l}\mathrm{CH}_{2}^{\mathrm{CH}_{2}-\mathrm{C}_{6} \mathrm{H}_{5}} \\
\mathrm{C}-\mathrm{CH}_{3} \mathrm{Cl}^{-}-\mathrm{CH}_{3} \\
\mathrm{R}=\mathrm{CH}_{2}\left(\mathrm{CH}_{2}\right)_{14} \mathrm{CH}_{3}\end{array}$ & & $\begin{array}{l}\text { Mouse: } L D_{50}=18 \mathrm{mg} / \mathrm{kg} \\
\quad \text { (intravenously) }\end{array}$ & {$[23]$} \\
\hline $\begin{array}{c}\text { Hexadecyl } \\
\text { benzyl dimethyl } \\
\text { ammonium chloride } \\
\mathrm{C}_{25} \mathrm{H}_{46} \mathrm{NCl}\end{array}$ & 396 & $\begin{array}{c}\mathrm{CH}_{2}-\mathrm{C}_{6} \mathrm{H}_{5} \\
\mathrm{CH}_{3}^{+}-\mathrm{CH}_{3} \\
\mathrm{Cl}=\mathrm{CH}_{2}\left(\mathrm{CH}_{2}\right)_{16} \mathrm{CH}_{3}\end{array}$ & & $\begin{array}{c}\text { Daphnia magna: } \\
E C_{50}=0.02 \mathrm{mg} / \mathrm{dm}^{3}(48 \mathrm{~h}) \\
\text { Algae: } I C_{50}=0.07 \mathrm{mg} / \mathrm{dm}^{3} \\
(72 \mathrm{~h}) \\
\text { Fish: } L C_{50}=0.31 \mathrm{mg} / \mathrm{dm}^{3} \\
(96 \mathrm{~h}) \\
\text { Rabbits: } L D_{50}=150 \mathrm{mg} / \mathrm{kg} \\
\text { (intravenously) }\end{array}$ & [24-26] \\
\hline
\end{tabular}

Table 4

Operating conditions of a chromatographic system during identification and quantitative determination of analytes from the cationic surfactants group in environmental samples

\begin{tabular}{|c|c|c|c|c|c|c|c|}
\hline Device type & \multicolumn{7}{|c|}{ Ion chromatograph ICS-3000 with autosampler } \\
\hline Analyte type & \multicolumn{3}{|c|}{ DTMA; BDDA-C12; HTMA; HP } & \multicolumn{4}{|c|}{ BDDA-C12; BDDA-C14; BDDA-C16 } \\
\hline Volume of dispensed sample $\left[\mathrm{cm}^{3}\right]$ & \multicolumn{7}{|c|}{0.010} \\
\hline Eluent composition & \multicolumn{3}{|c|}{$\begin{array}{c}\text { A: acetonitrile } \\
\text { B: } 20 \mathrm{mM} \text { acetic acid }\end{array}$} & \multicolumn{4}{|c|}{$\begin{array}{c}\text { A: deionised water, B: } 100 \mathrm{mN} \text { formic } \\
\text { acid, C: acetonitrile }\end{array}$} \\
\hline \multirow{5}{*}{ Elution gradient } & Time [min] & $\mathrm{A}$ & $\mathrm{B}$ & Time [min] & A & $\mathrm{B}$ & $\mathrm{C}$ \\
\hline & -3 & 20 & 80 & -7 & 20 & 15 & 65 \\
\hline & 0 & 20 & 80 & 0 & 20 & 15 & 65 \\
\hline & \multirow{2}{*}{11} & \multirow{2}{*}{70} & \multirow{2}{*}{30} & 10 & 50 & 15 & 35 \\
\hline & & & & 20 & 50 & 15 & 35 \\
\hline Mobile phase flow rate $\left[\mathrm{cm}^{3} / \mathrm{min}\right]$ & \multicolumn{3}{|c|}{0.30} & \multicolumn{4}{|c|}{0.25} \\
\hline $\begin{array}{c}\text { Analytical column (Column } \\
\text { diameter/length }[\mathrm{mm}])\end{array}$ & \multicolumn{7}{|c|}{ Acclaim $^{\circledR}$ Surfactant Plus $(2.1 / 150)$} \\
\hline Column temperature $\left[{ }^{\circ} \mathrm{C}\right]$ & \multicolumn{3}{|c|}{30} & \multicolumn{4}{|c|}{35} \\
\hline Suppressor type & \multicolumn{3}{|c|}{$\begin{array}{c}\text { CMMS } 300 \\
\text { (chemically regenerated with } \\
\text { a } 200 \mathrm{mM} \text { solution of potassium } \\
\text { hydroxide - flow } 1.2 \mathrm{~cm}^{3} / \mathrm{min} \text { ) }\end{array}$} & \multicolumn{4}{|c|}{$\begin{array}{l}\text { CSRS } 300 \\
\text { (regenerated with the use of external } \\
\text { deionised water - flow } 1.0 \mathrm{~cm}^{3} / \mathrm{min} \text { ) }\end{array}$} \\
\hline $\begin{array}{c}\text { Amperage during ion suppression } \\
{[\mathrm{mA}]}\end{array}$ & \multicolumn{3}{|c|}{ o } & \multicolumn{4}{|c|}{20} \\
\hline Detector type & \multicolumn{7}{|c|}{ conductometric } \\
\hline
\end{tabular}

DTMA - dodecyl trimethyl ammonium chloride, BDDA-C12 - benzyl dimethyl dodecyl ammonium chloride, HTMA - hexadecyl trimethyl ammonium chloride, HP - hexadecyl piridinium, BDDA-C14 - benzyl dimethyl tetradecyl ammonium chloride, BDDA-C16 - benzyl dimethyl hexadecyl ammonium chloride, CMMS - cation micro membrane suppressor, CSRS - cation self-regenerating suppressor 
As a result of chromatographic analysis chromatograms were obtained presenting the dependence of conductivity of certain analytes on the duration of analysis. Sample chromatograms of standard solutions obtained as a result of chromatographic analysis with the use of high performance ion chromatography - conductivity detection (HPIC-CD) system are presented at Figure 2 (according to the conditions presented in Table 4).

a)

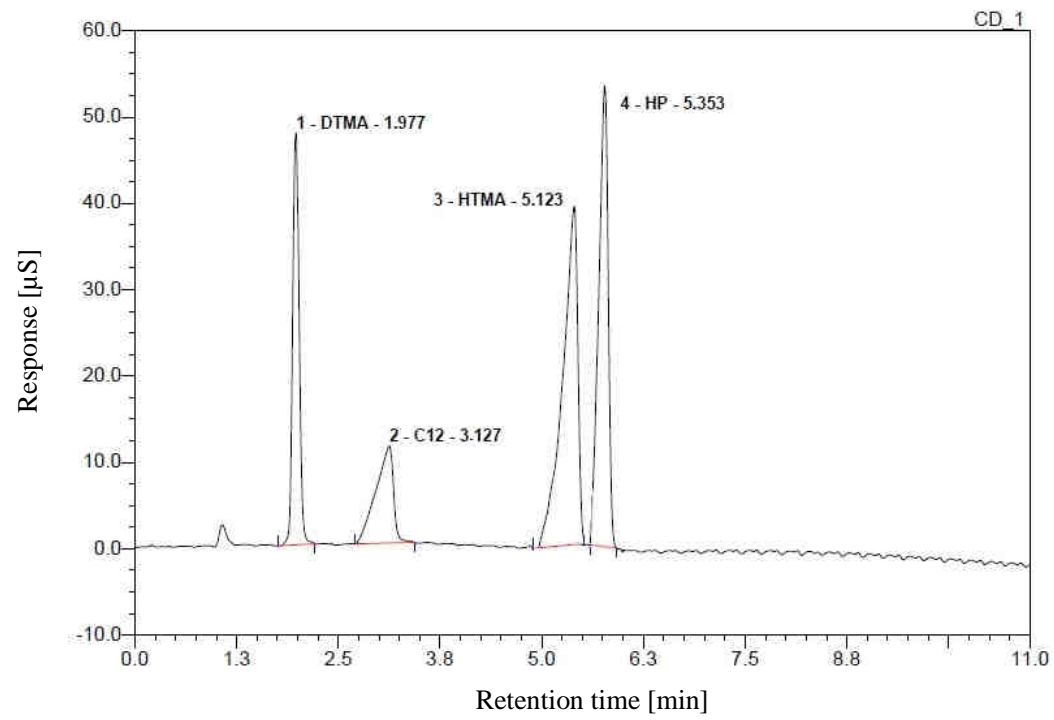

b)

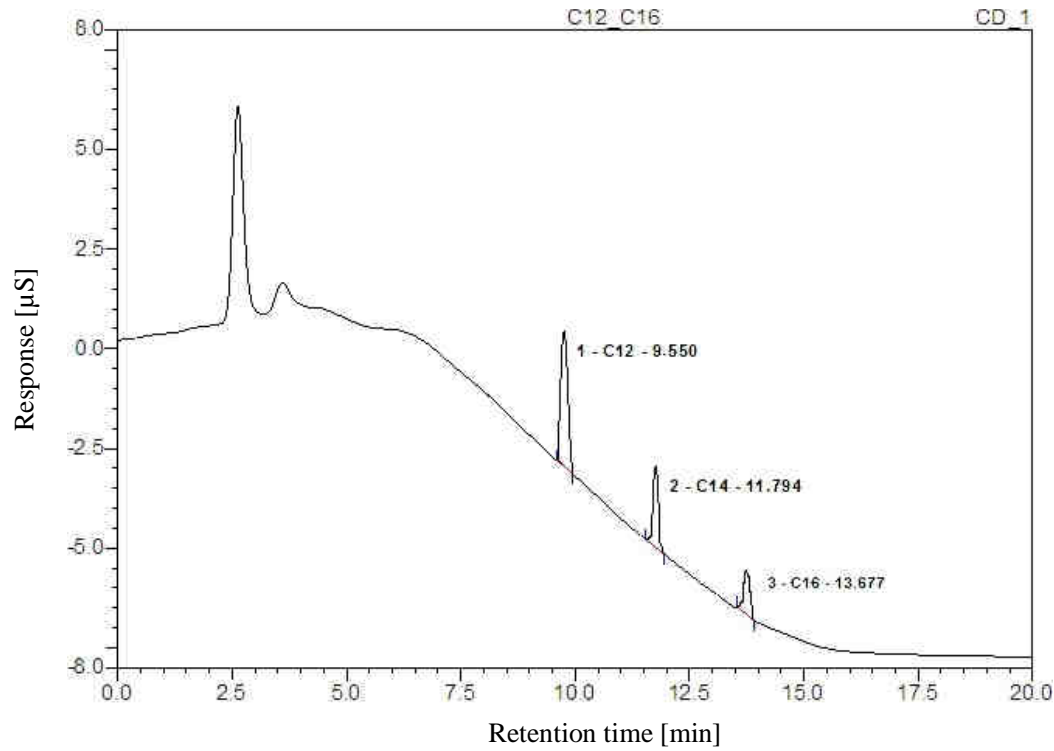

Fig. 2. Chromatograms obtained as a result of chromatographic analysis of samples of solutions containing a mixture of standard substances with the use of high performance ion chromatography-conductivity detection technique: a) concentration of analytes from the group of DTMA, BDDA-C12, HTMA, HP was $1000 \mu \mathrm{g} / \mathrm{dm}^{3}$; b) concentration of analytes from the group BDDA-C12, BDDA-C14, BDDA-C16 was $100 \mu \mathrm{g} / \mathrm{dm}^{3}$ 
Parameters of calibration curves created with the use of the HPIC-CD device as well as the calculated values of limit of detection (LOD) and limit of quantification (LOQ) for selected cationic surfactants are presented in Table 5.

Parameters of calibration curves for selected cationic surfactants

Table 5

\begin{tabular}{|c|c|c|c|c|c|c|}
\hline Analyte & $\begin{array}{c}\text { Analyte } \\
\text { retention time } \\
{[\mathrm{min}]}\end{array}$ & $\begin{array}{c}\text { Concentration } \\
\text { range } \\
{\left[\mu \mathrm{g} / \mathrm{dm}^{3}\right]}\end{array}$ & $\begin{array}{c}\text { Calibration curve } \\
\text { equation }\end{array}$ & $\begin{array}{l}\text { Regression } \\
\text { coefficient } r\end{array}$ & \begin{tabular}{|c|} 
Limit of \\
detection \\
$L O D$ \\
{$\left[\mu \mathrm{g} / \mathrm{dm}^{3}\right]$} \\
\end{tabular} & $\begin{array}{c}\text { Limit of } \\
\text { quantification } \\
L O Q \\
{\left[\mu \mathrm{g} / \mathrm{dm}^{3}\right]} \\
\end{array}$ \\
\hline \multirow{2}{*}{ DTMA } & \multirow{2}{*}{1.9} & $L O Q-50$ & $y=109 x+155$ & 0.9987 & \multirow{2}{*}{8.7} & \multirow[t]{2}{*}{$\begin{array}{lll}2 & 8 & 1\end{array}$} \\
\hline & & $50-1500$ & $y=173 x+34.5$ & 0.9998 & & \\
\hline \multirow{2}{*}{ BDDA-C12 } & \multirow{2}{*}{3.1} & $L O Q-50$ & $y=32.3 x-143$ & 0.9989 & \multirow{2}{*}{7.5} & \multirow{2}{*}{22} \\
\hline & & $50-1500$ & $y=37.2 x-123$ & 0.9992 & & \\
\hline \multirow{2}{*}{ HTMA } & \multirow{2}{*}{5.1} & $L O Q-50$ & $y=25.4 x-32.7$ & 0.9987 & \multirow{2}{*}{9.2} & \multirow{2}{*}{27} \\
\hline & & $50-1500$ & $y=23.8 x-45.8$ & 0.9989 & & \\
\hline \multirow{2}{*}{$\mathrm{HP}$} & \multirow{2}{*}{5.3} & $L O Q-50$ & $y=218 x-17.8$ & 0.9971 & \multirow{2}{*}{8.9} & \multirow{2}{*}{26} \\
\hline & & $50-1500$ & $y=278 x-23.1$ & 0.9983 & & \\
\hline \multirow{2}{*}{ BDDA-C12 } & \multirow{2}{*}{9.5} & $L O Q-50$ & $y=44.3 x+7.54$ & 0.9989 & \multirow{2}{*}{3.4} & \multirow{2}{*}{10} \\
\hline & & $50-1500$ & $y=49.1 x+6.45$ & 0.9993 & & \\
\hline \multirow{2}{*}{ BDDA-C14 } & \multirow{2}{*}{11.8} & $L O Q-50$ & $y=72.1 x+12.8$ & 0.9992 & \multirow{2}{*}{4.6} & \multirow{2}{*}{14} \\
\hline & & $50-1500$ & $y=77.5 x+11.5$ & 0.9993 & & \\
\hline \multirow{2}{*}{ BDDA-C16 } & \multirow{2}{*}{13.7} & $L O Q-50$ & $y=140 x+17.6$ & 0.9995 & \multirow{2}{*}{6.1} & \multirow{2}{*}{18} \\
\hline & & $50-1500$ & $y=163 x+32.2$ & 1 & & \\
\hline
\end{tabular}

The calculated values of selected validation parameters of the developed methodologies of determination of selected cationic surfactants in liquid and solid environmental samples are presented in Table 6.

Table 6

Values of selected validation parameters for proposed analytical methodologies determined with the use of ionic chromatography

\begin{tabular}{|c|c|c|c|c|c|c|}
\hline Analytes & $\begin{array}{c}\text { Range of } \\
\text { concentrations } \\
{\left[\mu \mathrm{g} / \mathbf{d m}^{3}\right]}\end{array}$ & $\begin{array}{c}\text { Calibration curve } \\
\text { equation }\end{array}$ & $\begin{array}{l}\text { Regression } \\
\text { coefficient } r\end{array}$ & $\begin{array}{c}\text { Method } \\
\text { detection } \\
\text { limit } M D L \\
{\left[\mu \mathrm{g} / \mathrm{dm}^{3}\right] \text { or }} \\
{[\mu \mathrm{g} / \mathrm{kg}]}\end{array}$ & \begin{tabular}{|c|} 
Method \\
quantification \\
limit $M Q L$ \\
{$\left[\mu \mathrm{g} / \mathrm{dm}^{3}\right]$ or } \\
{$[\mu \mathrm{g} / \mathrm{kg}]$} \\
\end{tabular} & $\begin{array}{l}\text { Analyte } \\
\text { recovery } \\
(C V[\%]) \\
(n=5)\end{array}$ \\
\hline \multirow{2}{*}{ DTMA } & \multirow{8}{*}{$M Q L-1000$} & $y=287 x+14.1$ & 0.9996 & 8.8 & 26 & $95.6(5.2)$ \\
\hline & & $y=408 x+34.2^{*}$ & $0.9990^{*}$ & $17^{*}$ & $42^{*}$ & $91.4(3.2)^{*}$ \\
\hline & & $y=148 x+87$ & 0.9983 & 8.6 & 25 & $83.4(0.3)$ \\
\hline C12 & & $y=276 x+47^{*}$ & $0.9981^{*}$ & $16^{*}$ & $41^{*}$ & $89.4(1.3)^{*}$ \\
\hline \multirow{2}{*}{ HTMA } & & $y=118 x+5.9$ & 0.9994 & 9.6 & 28 & $101.3(1.9)$ \\
\hline & & $y=199 x+14.3^{*}$ & $0.9995^{*}$ & $13^{*}$ & $39^{*}$ & $102.9(4.5)^{*}$ \\
\hline \multirow{2}{*}{ HP } & & $y=487 x+43.4$ & 0.9996 & 10 & 30 & $87.3(3.4)$ \\
\hline & & $y=687 x+18.1^{*}$ & $0.9989^{*}$ & $18^{*}$ & $48^{*}$ & $89.9(4.5)$ \\
\hline \multirow{3}{*}{$\begin{array}{c}\text { BDDA- } \\
\text { C12 }\end{array}$} & \multirow{7}{*}{$M Q L-1000$} & & & & & \\
\hline & & $y=1006 x+276$ & 0.9994 & 3.6 & 11 & $102.3(2.3)$ \\
\hline & & $y=1109 x+24.7^{*}$ & $0.9990^{*}$ & $7^{*}$ & $23^{*}$ & $99.2(2.9)^{*}$ \\
\hline \multirow{2}{*}{$\begin{array}{l}\text { BDDA- } \\
\text { C14 }\end{array}$} & & $y=827 x+11.7$ & 0.9999 & 4.3 & 13 & $104.2(1.6)$ \\
\hline & & $y=378 x+56.3^{*}$ & $0.9996^{*}$ & $11^{*}$ & $34^{*}$ & $78.9(3.7)^{*}$ \\
\hline \multirow{2}{*}{$\begin{array}{l}\text { BDDA- } \\
\text { C16 }\end{array}$} & & $y=674 x+39.5$ & 0.9997 & 6.8 & 20 & $81.9(3.6)$ \\
\hline & & $y=592 x+109^{*}$ & $0.9993^{*}$ & $17^{*}$ & $73^{*}$ & $89.1(7.4)^{*}$ \\
\hline
\end{tabular}

* selected parameters concern solid samples, $C V$ - coefficient of variation 


\section{Results}

The obtained results of determination of content levels of selected cationic surfactants with the use of the developed analytical procedures are presented in Tables 7 and 8.

Content levels of selected cationic surfactants determined in solid environmental samples

\begin{tabular}{|c|c|c|c|c|c|c|c|c|c|}
\hline \multirow{2}{*}{$\begin{array}{l}\text { Period of } \\
\text { sample } \\
\text { collection }\end{array}$} & \multirow{2}{*}{$\begin{array}{c}\text { Defined } \\
\text { parameter }\end{array}$} & \multirow{2}{*}{ DTMA } & \multirow{2}{*}{$\begin{array}{l}\text { BDDA- } \\
\text { C12 }\end{array}$} & \multirow{2}{*}{ HTMA } & \multirow{2}{*}{ HP } & \multirow{2}{*}{$\begin{array}{l}\text { BDDA- } \\
\text { C14 }\end{array}$} & \multirow{2}{*}{$\begin{array}{l}\text { BDDA- } \\
\text { C16 }\end{array}$} & \multicolumn{2}{|c|}{$\begin{array}{c}\text { Total SAC } \\
\text { content }\end{array}$} \\
\hline & & & & & & & & I & II \\
\hline \multicolumn{10}{|c|}{ SOLID SAMPLES $[\mu \mathrm{g} / \mathrm{kg}]$} \\
\hline \multirow{4}{*}{$\begin{array}{c}\text { Spring } \\
2012 \\
(n=11)\end{array}$} & $c_{\min } \pm U(k=2)$ & $<M D L$ & $\begin{array}{l}187.1 \\
\pm 8.9\end{array}$ & $210.6 \pm 7.3$ & $<M D L$ & $218.3 \pm 1.9$ & $284 \pm 10$ & \multirow{2}{*}{\multicolumn{2}{|c|}{$x \pm U(k=2)$}} \\
\hline & $c_{\max } \pm U(k=2)$ & $1798 \pm 11$ & $1198 \pm 29$ & $2014 \pm 10$ & $215 \pm 14$ & $1293 \pm 23$ & $1611 \pm 18$ & & \\
\hline & $\bar{C}$ & 342 & 464 & 610 & 87 & 515 & 654 & \multirow{2}{*}{$\begin{array}{l}2672 \\
\pm 207\end{array}$} & \multirow{2}{*}{$\begin{array}{r}2731 \\
\pm 318 \\
\end{array}$} \\
\hline & $S D$ & 529 & 281 & 580 & 96 & 294 & 443 & & \\
\hline \multirow{4}{*}{$\begin{array}{c}\text { Spring } \\
2013 \\
(n=15)\end{array}$} & $c_{\min } \pm U(k=2)$ & $<M D L$ & $<M D L$ & $53.0 \pm 4.5$ & $<M D L$ & 218 & 195 & \multirow{2}{*}{\multicolumn{2}{|c|}{$x \pm U(k=2)$}} \\
\hline & $c_{\max } \pm U(k=2)$ & $1216 \pm 13$ & $1198 \pm 10$ & $1338.3 \pm 9.8$ & $\begin{array}{c}244.5 \\
\pm 7.8 \\
\end{array}$ & $1285 \pm 11$ & $1599 \pm 12$ & & \\
\hline & $\bar{C}$ & 333 & 392 & 379 & 58 & 463 & 560 & \multirow{2}{*}{$\begin{array}{l}2184 \\
\pm 170 \\
\end{array}$} & \multirow{2}{*}{$\begin{array}{l}2206 \\
\pm 236 \\
\end{array}$} \\
\hline & $S D$ & 313 & 277 & 359 & 72 & 270 & 415 & & \\
\hline
\end{tabular}

$c_{\min ;} c_{\max }$ - the lowest and the highest determined concentration of analytes, respectively; $\bar{C}$ - arithmetic mean of the determined concentration of a given analyte in all samples collected in a given season; $S D$ - standard deviation; $x$ - overall concentration of analytes from the group of cationic analytes; Procedure I - accelerated solvent extraction + solid phase extraction + HPIC-CD; Procedure II - solid-liquid extraction + spectrophotometric technique

On the basis of the obtained results of the carried out research it may be stated that:

- content levels of certain analytes from the cationic surfactants group are within the range of the determined values of method quantification limit parameters up to $0.142 \pm 0.023 \mathrm{mg} / \mathrm{dm}^{3}$;

- the highest concentration of cationic analytes was found for "Summer 2012";

- maximum content levels of determined chemical compounds are within the range from $0.0896 \pm 0.0012$ (dodecyl trimethyl ammonium chloride) to $0.142 \pm 0.023 \mathrm{mg} / \mathrm{dm}^{3}$ (hexadecyl trimethyl ammonium chloride);

- average content levels of certain determined chemical compounds are within the range of $0.0105 \pm 0.093$ (HTMA) to $0.051 \pm 0.015 \mathrm{mg} / \mathrm{dm}^{3}$ (DTMA);

- the highest concentration levels of individual cationic analytes were determined for "Autumn 2011";

- in the same seasons in consecutive years a dropping tendency in contents of the determined compounds could be noticed (e.g. BDDA-C14, BDDA-C16) were not found in some surface water samples in the "Summer 2012" season and in previous seasons their presence was confirmed);

- in the case of overall contents of analytes from the cationic surfactants group obtained with the use of the elaborated analytical procedure and methodology recommended in the binding standard [27] similar levels of concentrations were determined (respectively $0.1076 \pm 0.0069-0.1590 \pm 0.0064 \mathrm{mg} / \mathrm{dm}^{3}$ and $\left.0.102 \pm 0.032-0.208 \pm 0.053 \mathrm{mg} / \mathrm{dm}^{3}\right)$;

- higher overall content levels of cationic analytes determined with the use of spectrophotometric and liquid-liquid extraction technique can be the result of 
co-extracting other substances with similar properties and of the type of the used technique at the stage of quantification (it is impossible to identify individual analytes).

Table 8

Levels of selected cationic surfactants determined in liquid environmental samples

\begin{tabular}{|c|c|c|c|c|c|c|c|c|c|}
\hline \multirow{2}{*}{$\begin{array}{l}\text { Period of } \\
\text { sample } \\
\text { collection }\end{array}$} & \multirow{2}{*}{$\begin{array}{c}\text { Defined } \\
\text { para- } \\
\text { meter }\end{array}$} & \multirow[t]{2}{*}{ DTMA } & \multirow{2}{*}{$\begin{array}{l}\text { BDDA- } \\
\text { C12 }\end{array}$} & \multirow[t]{2}{*}{ HTMA } & \multirow[t]{2}{*}{ HP } & \multirow{2}{*}{$\begin{array}{l}\text { BDDA- } \\
\text { C14 }\end{array}$} & \multirow{2}{*}{$\begin{array}{l}\text { BDDA- } \\
\text { C16 }\end{array}$} & \multicolumn{2}{|c|}{$\begin{array}{c}\text { Total SAC } \\
\text { content }\end{array}$} \\
\hline & & & & & & & & $\mathbf{I}$ & II \\
\hline \multicolumn{10}{|c|}{ LIQUID SAMPLES [mg/dm $\left.{ }^{3}\right]$} \\
\hline \multirow{4}{*}{$\begin{array}{c}\text { Autumn } \\
2011 \\
(n=19)\end{array}$} & $\begin{array}{c}c_{\min } \\
\pm U(k=2)\end{array}$ & \multicolumn{4}{|c|}{$<M D L$} & \multicolumn{2}{|c|}{$<M Q L$} & \multirow{2}{*}{\multicolumn{2}{|c|}{$x \pm U(k=2)$}} \\
\hline & $\begin{array}{c}c_{\max } \\
\pm U(k=2)\end{array}$ & $\begin{array}{c}0.0896 \\
\pm 0.0012\end{array}$ & $\begin{array}{c}0.0518 \\
\pm 0.0018\end{array}$ & $\begin{array}{c}0.0352 \\
\pm 0.0045\end{array}$ & $\begin{array}{c}0.0291 \\
\pm 0.0067\end{array}$ & $\begin{array}{c}0.0488 \\
\pm 0.0054\end{array}$ & $\begin{array}{c}0.0438 \\
\pm 0.0063\end{array}$ & & \\
\hline & $\bar{C}$ & 0.028 & 0.022 & 0.0105 & 0.0124 & 0.011 & 0.0237 & $\begin{array}{c}0.1076 \\
\pm\end{array}$ & $\begin{array}{c}0.150 \\
\pm\end{array}$ \\
\hline & $S D$ & 0.027 & 0.013 & 0.0093 & 0.0095 & 0.014 & 0.0087 & 0.0069 & 0.014 \\
\hline \multirow{4}{*}{$\begin{array}{c}\text { Winter } \\
2011 / 12 \\
(n=19)\end{array}$} & $\begin{array}{c}c_{\min } \\
\pm U(k=2)\end{array}$ & \multicolumn{5}{|c|}{$<M D L$} & MQL & \multirow{2}{*}{\multicolumn{2}{|c|}{$x \pm U(k=2)$}} \\
\hline & $\begin{array}{c}c_{\max } \\
\pm U(k=2)\end{array}$ & $\begin{array}{c}0.0885 \\
\pm 0.0045\end{array}$ & $\begin{array}{c}0.0487 \\
\pm 0.0034\end{array}$ & $\begin{array}{c}0.0498 \\
\pm 0.0014\end{array}$ & $\begin{array}{c}0.0274 \\
\pm 0.0023\end{array}$ & $\begin{array}{c}0.0516 \\
\pm 0.0017\end{array}$ & 0.049 & & \\
\hline & $\bar{C}$ & 0.042 & 0.021 & 0.011 & 0.012 & 0.011 & 0.024 & $\begin{array}{c}0.1214 \\
\pm\end{array}$ & $\begin{array}{c}0.102 \\
\pm\end{array}$ \\
\hline & $S D$ & 0.027 & 0.011 & 0.011 & 0.0057 & 0.015 & 0.012 & 0.0817 & 0.032 \\
\hline \multirow{4}{*}{$\begin{array}{c}\text { Spring } \\
2012 \\
(n=19)\end{array}$} & $\begin{array}{c}c_{\min } \\
\pm U(k=2)\end{array}$ & $\begin{array}{c}0.0017 \\
\pm 0.0010\end{array}$ & $<M Q L$ & \multicolumn{2}{|c|}{$<M D L$} & \multicolumn{2}{|c|}{$<M Q L$} & \multirow{2}{*}{\multicolumn{2}{|c|}{$x \pm U(k=2)$}} \\
\hline & $\begin{array}{c}c_{\max } \\
\pm U(k=2)\end{array}$ & $\begin{array}{c}0.0844 \\
\pm 0.0035 \\
\end{array}$ & $\begin{array}{r}0.0753 \\
\pm 0.0018 \\
\end{array}$ & $\begin{array}{c}0.0960 \\
\pm 0.0025 \\
\end{array}$ & $\begin{array}{c}0.0215 \\
\pm 0.0035\end{array}$ & $\begin{array}{c}0.0527 \\
\pm 0.0065\end{array}$ & $\begin{array}{c}0.0591 \\
\pm 0.0038\end{array}$ & & \\
\hline & $\bar{C}$ & 0.028 & 0.025 & 0.015 & 0.0115 & 0.012 & 0.025 & $\begin{array}{c}0.1165 \\
\pm\end{array}$ & $\begin{array}{c}0.1648 \\
\pm\end{array}$ \\
\hline & $S D$ & 0.020 & 0.016 & 0.019 & 0.0070 & 0.016 & 0.013 & 0.0047 & 0.0025 \\
\hline \multirow{4}{*}{$\begin{array}{c}\text { Summer } \\
2012 \\
(n=19)\end{array}$} & $\begin{array}{c}c_{\min } \\
\pm U(k=2)\end{array}$ & \multicolumn{6}{|c|}{$<M D L$} & \multirow{2}{*}{\multicolumn{2}{|c|}{$x \pm U(k=2)$}} \\
\hline & $\begin{array}{c}c_{\max } \\
\pm U(k=2)\end{array}$ & $\begin{array}{c}0.0618 \\
\pm 0.0044\end{array}$ & $\begin{array}{c}0.0434 \\
\pm 0.0028\end{array}$ & $\begin{array}{c}0.142 \\
\pm 0.023\end{array}$ & $\begin{array}{c}0.0335 \\
\pm 0.0025\end{array}$ & $\begin{array}{c}0.0453 \\
\pm 0.0094\end{array}$ & $\begin{array}{c}0.0651 \\
\pm 0.0015\end{array}$ & & \\
\hline & $\bar{C}$ & 0.024 & 0.023 & 0.018 & 0.0180 & 0.012 & 0.030 & $\begin{array}{c}0.1250 \\
\pm\end{array}$ & $\begin{array}{c}0.208 \\
\pm\end{array}$ \\
\hline & $S D$ & 0.018 & 0.013 & 0.029 & 0.0094 & 0.015 & 0.014 & 0.0068 & 0.053 \\
\hline \multirow{4}{*}{$\begin{array}{c}\text { Autumn } \\
2012 \\
(n=40)\end{array}$} & $\begin{array}{c}c_{\min } \\
\pm U(k=2)\end{array}$ & \multicolumn{6}{|c|}{$<M D L$} & \multirow{2}{*}{\multicolumn{2}{|c|}{$x \pm U(k=2)$}} \\
\hline & $\begin{array}{c}c_{\max } \\
\pm U(k=2)\end{array}$ & $\begin{array}{c}0.0840 \\
\pm 0.0030 \\
\end{array}$ & $\begin{array}{c}0.0991 \\
\pm 0.0022 \\
\end{array}$ & $\begin{array}{c}0.0506 \\
\pm 0.0011 \\
\end{array}$ & $\begin{array}{c}0.1160 \\
\pm 0.0024\end{array}$ & $\begin{array}{c}0.0761 \\
\pm 0.0033\end{array}$ & $\begin{array}{c}0.0886 \\
\pm 0.0024\end{array}$ & & \\
\hline & $\bar{C}$ & 0.051 & 0.029 & 0.012 & 0.017 & 0.022 & 0.028 & $\begin{array}{c}0.1590 \\
+\end{array}$ & $\begin{array}{c}0.187 \\
+\end{array}$ \\
\hline & $S D$ & 0.015 & 0.027 & 0.013 & 0.029 & 0.019 & 0.020 & 0.0024 & 0.014 \\
\hline \multirow{4}{*}{$\begin{array}{c}\text { Winter } \\
2012 / 13 \\
(n=40)\end{array}$} & $\begin{array}{c}c_{\min } \\
\pm U(k=2)\end{array}$ & \multicolumn{6}{|c|}{$<M D L$} & \multirow{2}{*}{\multicolumn{2}{|c|}{$x \pm U(k=2)$}} \\
\hline & $\begin{array}{c}c_{\max } \\
\pm U(k=2)\end{array}$ & $\begin{array}{c}0.0525 \\
\pm 0.0026\end{array}$ & $\begin{array}{c}0.0983 \\
\pm 0.0016\end{array}$ & $\begin{array}{c}0.0498 \\
\pm 0.0044\end{array}$ & $\begin{array}{c}0.0312 \\
\pm 0.0066\end{array}$ & $\begin{array}{c}0.0550 \\
\pm 0.0032\end{array}$ & $\begin{array}{c}0.0645 \\
\pm 0.0011\end{array}$ & & \\
\hline & $\bar{C}$ & 0.026 & 0.020 & 0.013 & 0.011 & 0.018 & 0.019 & $\begin{array}{c}0.1070 \\
\pm\end{array}$ & $\begin{array}{c}0.105 \\
\pm\end{array}$ \\
\hline & $S D$ & 0.012 & 0.015 & 0.013 & 0.0072 & 0.014 & 0.010 & 0.0029 & 0.017 \\
\hline
\end{tabular}




\begin{tabular}{|c|c|c|c|c|c|c|c|c|c|}
\hline \multirow{2}{*}{$\begin{array}{l}\text { Period of } \\
\text { sample } \\
\text { collection }\end{array}$} & \multirow{2}{*}{$\begin{array}{c}\text { Defined } \\
\text { para- } \\
\text { meter }\end{array}$} & \multirow[t]{2}{*}{ DTMA } & \multirow{2}{*}{$\begin{array}{l}\text { BDDA- } \\
\text { C12 }\end{array}$} & \multirow[t]{2}{*}{ HTMA } & \multirow[t]{2}{*}{ HP } & \multirow{2}{*}{$\begin{array}{c}\text { BDDA- } \\
\text { C14 }\end{array}$} & \multirow{2}{*}{$\begin{array}{c}\text { BDDA- } \\
\text { C16 }\end{array}$} & \multicolumn{2}{|c|}{$\begin{array}{c}\text { Total SAC } \\
\text { content }\end{array}$} \\
\hline & & & & & & & & $\mathbf{I}$ & II \\
\hline \multicolumn{10}{|c|}{ LIQUID SAMPLES [mg/dm $\left.{ }^{3}\right]$} \\
\hline \multirow{4}{*}{$\begin{array}{c}\text { Spring } \\
2013 \\
(n=40)\end{array}$} & $\begin{array}{c}c_{\min } \\
\pm U(k=2)\end{array}$ & \multicolumn{2}{|c|}{$<M D L$} & $<M Q L$ & \multicolumn{3}{|c|}{$<M D L$} & \multirow{2}{*}{\multicolumn{2}{|c|}{$x \pm U(k=2)$}} \\
\hline & $\begin{array}{c}c_{\max } \\
\pm U(k=2) \\
\end{array}$ & $\begin{array}{c}0.0435 \\
\pm 0.0015 \\
\end{array}$ & $\begin{array}{c}0.0893 \\
\pm 0.0023 \\
\end{array}$ & $\begin{array}{c}0.0374 \\
\pm 0.0010 \\
\end{array}$ & $\begin{array}{c}0.0994 \\
\pm 0.0042\end{array}$ & $\begin{array}{c}0.0715 \\
\pm 0.0022\end{array}$ & $\begin{array}{c}0.0894 \\
\pm 0.0061\end{array}$ & & \\
\hline & $\bar{C}$ & 0.023 & 0.021 & 0.0171 & 0.017 & 0.019 & 0.022 & $\begin{array}{c}\text { 0.1191 } \\
\pm\end{array}$ & $\begin{array}{c}0.152 \\
\pm\end{array}$ \\
\hline & $S D$ & 0.012 & 0.018 & 0.0094 & 0.016 & 0.017 & 0.015 & 0.0033 & 0.029 \\
\hline
\end{tabular}

$c_{\min ;} c_{\max }$ - the lowest and the highest determined concentration of analytes, respectively; $\bar{C}$ - arithmetic mean of the determined concentration of a given analyte in all samples collected in a given season; $S D$ - standard deviation; Procedure I - solid phase extraction + HPIC-CD; Procedure II - liquid-liquid extraction (LLE) + spectrophotometric technique

Figure 3 presents data concerning the dependence of content levels of the determined cationic analytes in samples of bottom sediments as regards their place of collection. On the basis of the obtained results we may state that solid samples marked with the numbers 5 and 16 have been collected in areas with the highest pollution level in a given ecosystem. Besides, higher concentrations of analytes containing longer alkyl chains in their structure were determined. Hydrophobic properties of the mentioned chemical compounds increase their affinity to undergo sorption processes on the surface of solids.

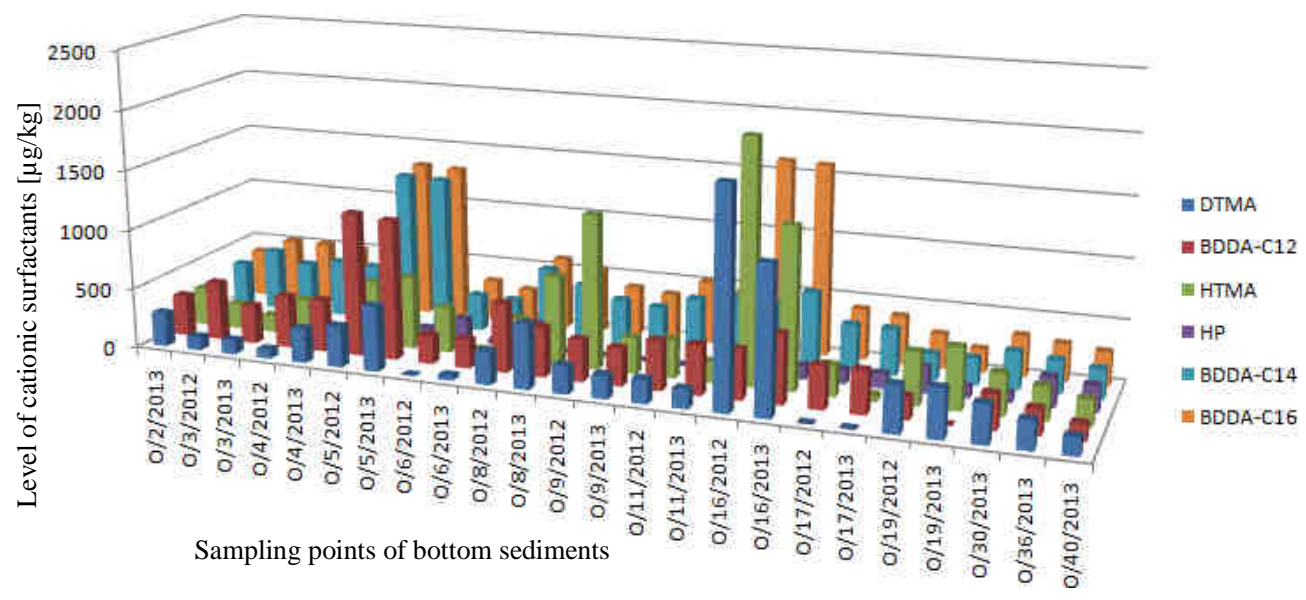

Fig. 3. Content levels of cationic surfactants determined in samples of bottom sediments collected from different places within the studied ecosystem in the years 2012 and 2013

Figure 4 presents details concerning the dependence between concentration levels of individual determined surfactants in liquid and solid samples collected from the same areas in the river catchment (percentage). Comparing the relationship between the obtained percentage of content of certain cationic analytes with overall concentration levels determined in environmental samples, it can be stated that these relationships are similar for most batches of tested samples and in most samples no hexadecyl piridinium compound was found. Only solid samples marked 16 and 19 contain respectively 1 and $10 \%$ of HP, 
what may be the evidence of a point source of contamination of this ecosystem area. Samples of bottom deposits marked as 9 and 19 have the highest content of HTMA expressed as a percentage. However, in liquid samples collected from the area marked with 9 (area of the mouth of the Slepotka river flowing into the Klodnica) the highest percentage concentrations of the DTMA surfactant have been found and TMA group surfactants have also been detected in these samples.

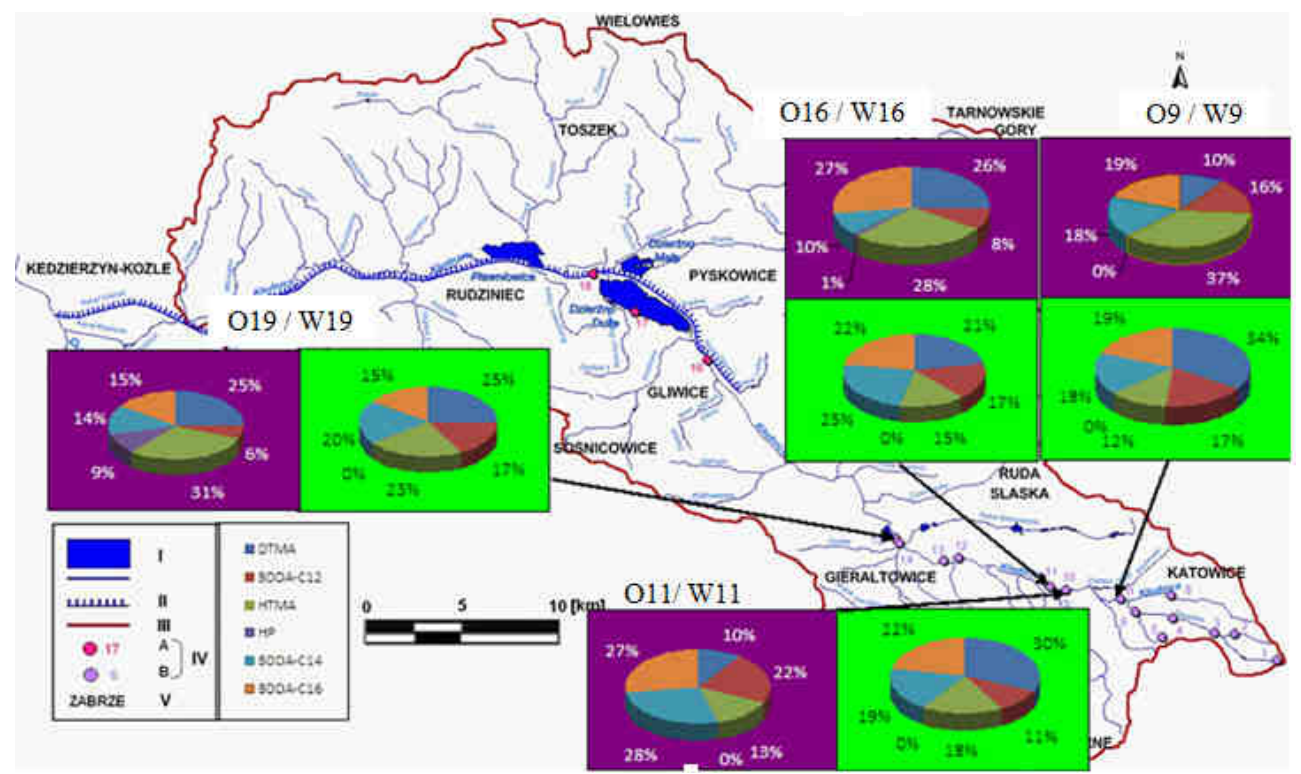

Fig. 4. The relationship between concentration levels of certain surfactants and their overall content in liquid and solid samples collected in the same areas in the studied ecosystem (green colour water samples collected in the period 2010/2012 and 2013; purple colour - samples of deposits collected in 2012 and 2013)

In the literature, there are described only few studies concerning compounds from the group of QAC marked in environmental samples (Table 9 and [28]). Few studies reported that levels of QAC in surface waters are in the range of 1.8 to $19 \mathrm{ng} / \mathrm{dm}^{3}$. In the samples taken from the Klodnica River these values are significantly higher and range from 1 to $51 \mu \mathrm{g} / \mathrm{dm}^{3}$. Determined levels of cationic surfactants are similar to the results obtained for the river water described by Wee and Kennedy (5 to $30 \mu \mathrm{g} / \mathrm{dm}^{3}$ ) [29]. Generally, QAC contamination of Klodnica sediments was also higher comparing to other investigated areas [30, 31]. For the Klodnica River these values may be higher because the investigated river runs through highly urbanised (with different populations) and industrial areas. Occurrence of SAC, which toxicity is still unknown also as mixtures with other pollutants, in environmental samples indicates that conventional processes in wastewater treatment plants are not able to remove completely surfactants but also they can illegally be emitted into river catchment. The emission of investigated compounds into environmental ecosystems can lead to serious problems associated with higher ecological and human health risk [32]. 
Determination of individual QAC in environmental samples

\begin{tabular}{|c|c|c|c|c|}
\hline Type of sample/origin & Determined substance & \multicolumn{2}{|c|}{ Concentration ranges } & References \\
\hline \multirow[t]{2}{*}{ Roof runoff } & \multirow[t]{3}{*}{$\mathrm{BAC}(\mathrm{C}-12+\mathrm{C}-14)$} & \begin{tabular}{|c|} 
Sum of dissolved \\
SAC + \\
particles \\
{$\left[\mu \mathrm{g} / \mathrm{dm}^{3}\right]$}
\end{tabular} & $\begin{array}{c}\text { Dissolved } \\
\text { fraction of SAC } \\
{\left[\mu \mathrm{g} / \mathrm{dm}^{3}\right]}\end{array}$ & \multirow[t]{3}{*}[33,34]{} \\
\hline & & $27-5525$ & $22-3739$ & \\
\hline Stormwater samples & & $7-28$ & $0.1-0.3$ & \\
\hline Seawater (England) & \multirow{4}{*}{ DADMAC C-10 } & \multicolumn{2}{|c|}{$\left[\mathrm{ng} / \mathrm{dm}^{3}\right]$} & \multirow{4}{*}{ [35] } \\
\hline Tynemouth & & $120-$ & -270 & \\
\hline Cullercoats & & 13 & & \\
\hline St Mary's & & $\mathrm{N}$ & & \\
\hline \multirow{2}{*}{ Surface water } & ATMAC (C12-16) & 4- & & {$[10]$} \\
\hline & $\mathrm{BAC}(\mathrm{C} 12-\mathrm{C} 16)$ & 1.8 & -38 & {$[25]$} \\
\hline \multirow{3}{*}{ River water } & ATMAC (C12-C18) & $\leq$ & 10 & {$[36]$} \\
\hline & DTDMAC & $5000-3$ & 30000 & \multirow[b]{2}{*}{ [29] } \\
\hline & $\begin{array}{l}\text { STEDBAC, HDPC, } \\
\text { DOTMAC }\end{array}$ & \multicolumn{2}{|c|}{$<2000$} & \\
\hline Urban estuarine sediments & $\begin{array}{c}\text { BAC, ATMAC, } \\
\text { DADMAC }\end{array}$ & \multicolumn{2}{|c|}{$\begin{array}{c}49.3-1050,16.5-2010 \\
159-7700 \mathrm{ng} / \mathrm{g}\end{array}$} & {$[30]$} \\
\hline $\begin{array}{c}\text { Sediment (Faroe Islands/ } \\
\text { Finland/Greenland/Norway/Sweden) }\end{array}$ & $\begin{array}{c}\text { ATAC (C12-22), BAC } \\
\text { (C12-18), DDAC } \\
(\mathrm{C} 10-18)\end{array}$ & \multicolumn{2}{|c|}{$\begin{array}{l}<0.35-450,0.17-1200 \\
<0.055-12000 \mathrm{ng} / \mathrm{g}\end{array}$} & {$[31]$} \\
\hline
\end{tabular}

BAC - benzyl ammonium chloride, DADMAC/DDAC - dialkyldimethylammonium compounds, ATMAC/ATAC - alkil trimethyl ammonium chloride, STEDBAC - stearyldimethyl benzyl ammonium chloride, HDPC - 1-hexadecyl pyridinium chloride, DOTMAC - dodecyl trimethyl ammonium chloride, $N D$ - not detected

\section{Conclusions}

The developed analytical procedures have been used to determine content levels of selected cationic surfactants in surface water samples ( 200 samples) and bottom sediment samples (15 samples). In most samples analytes from the cationic SAC group were found (areas where samples were collected are under heavy anthropogenic impact). The determined concentration levels were in the range below the determined values of $M D L$ or $M Q L$ up to $0.142 \pm 0.023 \mathrm{mg} / \mathrm{dm}^{3}$ or $2014 \pm 10 \mu \mathrm{g} / \mathrm{kg}$ (liquid and solid samples, respectively).

Comparing the concentrations of certain analytes found in liquid and solid environmental samples, it can be noticed that the surfactants containing a shorter alkyl chain in a molecule were present in higher concentrations in liquid samples (hydrophobicity increasing with the increasing length of the chain). Alkyl benzyl dimethyl ammonium and HTMA compounds may undergo sorption processes on the surface of solid particles of bottom deposits so their low content levels were determined.

\section{Acknowledgments}

This work has been financially supported by the Polish Ministry of Science and Higher Education (project ST - 02-0103/07 and MN - 01-0211/08/322). 


\section{References}

[1] Olkowska E, Ruman M, Polkowska Ż. Anal Methods Chem. 2014;ID 769708. DOI: 10.1155/2014/769708.

[2] Merretting-Bruns U, Jelen E. Materials. 2009;2:181-206. DOI: 10.3390/ma2010181.

[3] Knezovich JP, Lawton MP, Inouye LS. Bull Environ Contam Toxicol. 1989;42:87-93. DOI: 10.1007/BF01699208.

[4] Gerba CP. Quaternary ammonium biocides: efficacy in application. Appl Environ Microbiol. 2015;81:464-469. DOI:10.1128/AEM.02633-14.

[5] Sütterlin H, Alexy R, Coker A, Kümmerer K. Chemosphere. 2008;72:479-484. DOI: 10.1016/j.ecoenv.2007.12.015.

[6] Zhang C, Tezel U, Li K, Liu D, Ren R, Du J, et al. Water Res. 2011;45:1238-1246. DOI: 10.1016/j.watres.2010.09.037.

[7] Garcia MT, Ribosa I, Guindulain T, Sanchez-Leal J, Vives-Rego J. Environ Pollut. 2001;111:169-175. DOI: 10.1016/S0269-7491(99)00322-X.

[8] Swisher RD. Surfactant Biodegradation, vol. 18 of Surfactant Science Series. New York: Marcel Dekker; 1987. ISBN: 9780824769383.

[9] Boethling RS. Water Res. 1984;18:1061-1076. DOI: 10.1016/0043-1354(84)90220-3.

[10] Martinez-Carballo E, Sitka A, Gonzalez-Barreiro C, Kreuzinger N, Furhacker M, Scharf S, et al. Environ Pollut. 2007;145:489-496. DOI: 10.1016/j.envpol.2006.04.033.

[11] Tezel U, Pierson JA, Pavlostathis SG. Water Res. 2006;40:3660-3668. DOI:10.1016/j.watres.2006.06.019.

[12] Utsunomiya A, Watanuki T, Matsushita K, Nishina M, Tomita I. Chemosphere. 1997;35:2479-2490. DOI: 10.1016/S0045-6535(97)00316-0.

[13] Lewis M, Wee V. Environ Toxicol Chem. 1983;2:105-118. DOI: 10.1002/etc.5620020112.

[14] Singh RP, Gupta N, Singh S, Singh A, Suman R, Annie K. Bull Environ Contam Toxicol. 2002;69:265-270. DOI : 10.1007/s00128-002-0056-z.

[15] Ruman M, Olkowska E, Kozioł K, Absalon D, Matysik M, Polkowska Ż. J Environ Qual. 2014;43:753-762. DOI: $10.2134 /$ jeq2013.06.0225.

[16] Olkowska E, Kudłak B, Tsakovski S, Ruman M, Simeonov V, Polkowska Ż. Sci Total Environ. 2014;476-477:477-484. DOI: 10.2478/eces-2013-0033.

[17] http://www.przyjaznaklodnica.pl/dokumenty/Fakty/Mapa_zlewni_Klodnicy.jpg

[18] Ruman M, Olkowska E, Drąg M, Jankowski G, Polkowska Ż. Ecol Chem Eng S. 2017;24(1):53-63, DOI: 10.1515/eces-2017-0005.

[19] Olkowska E, Polkowska Ż, Namieśnik J. Talanta. 2013;116:210-216. DOI: 10.1016/j.talanta.2011.10.034

[20] Olkowska E, Ruman M, Kowalska A, Polkowska Ż. Ecol Chem Eng S. 2013;20(2):331-342. DOI: 10.2478/eces-2013-0024.

[21] Marhold J. Prehled Prumyslove Toxikologie. Organicke Latky. (Industrial Toxicology Overview. Organic Lattice.). Prague: Avicenum; 1986. ISBN: 0805986.

[22] http://chem.sis.nlm.nih.gov/chemidplus/jsp/common/Toxicity.jsp.

[23] http://www.sigmaaldrich.com/catalog/ProductDetail.do?lang=en\&N4=13401|FLUKA\&N5 $=$ SEARCH_CONCAT_PNO|BRAND_KEY\&F=SPECi.

[24] http://www.chemcas.com/msds/cas/msds8/122-18-9.asp.

[25] http://www.sigmaaldrich.com/catalog/ProductDetail.do?lang=pl\&N4=B4136|SIGMA\&N5 $=$ SEARCH_CONCAT_PNO|BRAND_KEY\&F=SPEC.

[26] Schardein JL. Antimicrobial Agents. Chemically Induced Birth Defects. New York: Marcel Dekker; 1993. ISBN 10: 0824787757.

[27] DIN 38409-20 Deutsche Einheitsverfahren zur Wasser-, Abwasser- und Schlammuntersuchung; Summarische Wirkungs- und Stoffkenngrößen (Gruppe $\mathrm{H}$ ); Bestimmung der disulfinblau-aktiven Substanzen (H 20) (German standard methods for the examination of water, waste water and sludge; parameters characterizing effects and substances (group $\mathrm{H}$ ); determination of substances that react with disulfine blue (H 20)). German Institute for Standardization; 1989. https://www.beuth.de/en/standard/din38409-20/1443160.

[28] Zhang C, Cui F, Zeng GM, Jiang M, Yang ZZ, Yu ZG et al. Sci Total Environ. 2015;518-519:352-62. DOI: 10.1016/j.scitotenv.2015.03.007.

[29] Wee VT, Kennedy JM. Anal Chem. 1982;54:1631-1633. DOI: 10.1021/ac00246a037.

[30] Li X, Luo X, Mai B, Liu J, Chen L, Lin S. Environ Pollut. 2014;185:127-133. DOI: 10.1016/j.envpol.2013.10.028.

[31] Kaj L, Wallberg P, Brorström-Lundén E. Quaternary ammonium compounds, Analyses in a Nordic cooperation on screening. TemaNord 2014:556. http://norden.diva-portal.org/smash/get/ diva2:760465/FULLTEXT01.pdf. ISBN 978-92-893-3815-8. 
[32] Jackson M, Eadsforth C, Schowanek D, Delfosse T, Riddle A, Budgen N. Environ Toxicol Chem. 2016;35:1077-1086. DOI: 10.1002/etc.3297.

[33] Van de Voorde A, Lorgeoux C, Gromaire MCh, Chebbo G. Environ Pollut. 2012;164:150-157, DOI:10.1016/j.envpol.2012.01.037.

[34] Grillitsch B, Gans O, Kreuzinger N, Scharf S, Uhl M, Fuerhacker M. Environ Water Sci Technol. 2006;54:111-118. DOI: 10.2166/wst.2006.840.

[35] Bassarab P, Williams D, Dean JR, Ludkin E, Perry JJ. J Chromatogr A. 2011;1218:673-677. DOI: 10.1016/j.chroma.2010.11.088

[36] Ding WH, Tsai PC. Anal Chem. 2003;75:1792-1797. DOI: 10.1021/ac020536y. 